\title{
Explicit General Linear Methods with a Large Stability Region for Volterra Integro-differential Equations
}

\section{Hassan Mahdi, Gholamreza Hojjati and Ali Abdi}

Faculty of Mathematical Sciences, University of Tabriz

29 Bahman Blvd, Tabriz, Iran

E-mail: h.mahdi@tabrizu.ac.ir

E-mail: ghojjati@tabrizu.ac.ir

E-mail(corresp.): a_abdi@tabrizu.ac.ir

Received December 21, 2018; revised May 23, 2019; accepted May 23, 2019

\begin{abstract}
In this paper, we describe the construction of a class of methods with a large area of the stability region for solving Volterra integro-differential equations. In the structure of these methods which is based on a subclass of explicit general linear methods with and without Runge-Kutta stability property, we use an adequate quadrature rule to approximate the integral term of the equation. The free parameters of the methods are used to obtain methods with a large stability region. The efficiency of the proposed methods is verified with some numerical experiments and comparisons with other existing methods.
\end{abstract}

Keywords: Volterra integro-differential equations, general linear methods, Runge-Kutta stability, region of absolute stability, Gregory quadrature rule.

AMS Subject Classification: 65L05; 65R20; 65L20.

\section{Introduction}

In this paper we deal with the numerical solution of Volterra integro-differential equations (VIDEs) of the form

$$
\left\{\begin{array}{l}
y^{\prime}(t)=f(t, y(t), z(t)), \quad t \in I:=\left[t_{0}, T\right] \\
y\left(t_{0}\right)=y_{0}
\end{array}\right.
$$

with $z(t):=\int_{t_{0}}^{t} K(t, s, y(s)) d s$. Here, the functions $f: I \times \mathbb{R}^{m} \times \mathbb{R}^{m} \rightarrow \mathbb{R}^{m}$ and $K: D \times \mathbb{R}^{m} \rightarrow \mathbb{R}^{m}$, with $D:=\left\{(t, s): t_{0} \leq s \leq t \leq T\right\}$ are continuous on 
their respective domain and $m$ representing the dimensionality of the system. Also, $f$ satisfies the Lipschitz conditions with respect to $y$ and $z$ whereas $K$ satisfies the Lipschitz condition with respect to $y$. These assumptions guarantee the existence and uniqueness of the sufficiently smooth solution [29]. Many medical science, biology, and physical problems can be modeled in the form of VIDEs [10]. Recently, VIDEs have attracted the interest of many scientists and researchers due to their wide range of applications in science and technology and many numerical methods have been proposed. Using efficient numerical methods for initial value problems (IVPs) in ordinary differential equations (ODEs) has been successfully studied to design methods for the numerical solution of VIDE (1.1). Linz [29] has extended the linear multistep methods and has given a convergence theorem analogous to the one holding for ODEs. Brunner and Lambert [9] and Matthys [33] have examined the stability properties of these methods. Makroglou [32] has extended the theory of hybrid methods for the numerical solution of (1.1). Special Runge-Kutta methods for VIDEs have been presented by Wolfe and Phillips [36], Lubich [30], and Brunner [7].

Furthermore, collocation and spectral collocation methods have been studied in $[8,10,20,21,22,26,27,35]$. Also, an elegant numerical method based on linear barycentric rational interpolation has been introduced by Abdi and Hosseini [1]. Recently, a method based on general linear methods (GLMs) $[11,13,28]$ for the numerical solution of (1.1) has been introduced and studied by Mahdi et al. in [31].

In this paper, we introduce explicit methods for the numerical solution of VIDE (1.1) based on diagonally implicit multistage integration methods (DIMSIMs) as a subclass of GLMs. DIMSIMs for the numerical solution of ODEs

$$
\left\{\begin{array}{l}
y^{\prime}(t)=f(t, y(t)), \quad t \in\left[t_{0}, T\right] \\
y\left(t_{0}\right)=y_{0}
\end{array}\right.
$$

were introduced by Butcher [12] and extended by Butcher and Jackiewicz [14, $15,16,17]$. These methods are characterized by four integers $(p, q, r, s)$ and four coefficients matrices

$$
A=\left[a_{i j}\right] \in \mathbb{R}^{s \times s}, \quad U=\left[u_{i j}\right] \in \mathbb{R}^{s \times r}, \quad B=\left[b_{i j}\right] \in \mathbb{R}^{r \times s}, \quad V=\left[v_{i j}\right] \in \mathbb{R}^{r \times r},
$$

where $p$ and $q$ are respectively the order and the stage order of the method, $r$ is the number of input and output approximations, and $s$ is the number of internal stages. Let $Y^{[n]}=\left[Y_{i}^{[n]}\right]_{i=1}^{s}$ be an approximation of the stage order $q$ to the vector $y\left(t_{n-1}+c h\right)=\left[y\left(t_{n-1}+c_{i} h\right)\right]_{i=1}^{s}$ where $c=\left[\begin{array}{llll}c_{1} & c_{2} & \cdots & c_{s}\end{array}\right]^{T} \in \mathbb{R}^{s}$ designates the abscissa vector and $f\left(Y^{[n]}\right)=\left[f\left(Y_{i}^{[n]}\right)\right]_{i=1}^{s}$ denotes the first derivative stage value vector. The external stage $y^{[n]}=\left[y_{i}^{[n]}\right]_{i=1}^{r}$ is defined as a $p$-th order approximation of the linear combinations of the derivatives, i.e.,

$$
y^{[n]}=q_{0} y\left(x_{n}\right)+q_{1} h y^{\prime}\left(x_{n}\right)+q_{2} h^{2} y^{\prime \prime}\left(x_{n}\right)+\cdots+q_{p} h^{p} y^{(p)}\left(x_{n}\right)+\mathcal{O}\left(h^{p+1}\right),
$$

for some real vectors $q_{i} \in \mathbb{R}^{r}, i=0,1, \ldots, p$ (see [13,28]). A DIMSIM, used for 
the numerical solution of ODEs (1.2), is defined by

$$
\left\{\begin{array}{l}
Y_{i}^{[n]}=h \sum_{j=1}^{s} a_{i j} f\left(t_{n-1}+c_{j} h, Y_{j}^{[n]}\right)+\sum_{j=1}^{r} u_{i j} y_{j}^{[n-1]}, i=1,2, \ldots, s, \\
y_{i}^{[n]}=h \sum_{j=1}^{s} b_{i j} f\left(t_{n-1}+c_{j} h, Y_{j}^{[n]}\right)+\sum_{j=1}^{r} v_{i j} y_{j}^{[n-1]}, \quad i=1,2, \ldots, r
\end{array}\right.
$$

where $n=1,2, \ldots, N, N h=T-t_{0}, h$ is the stepsize, and $t_{n}=t_{0}+n h$. These methods have been divided into four types [28]. The coefficients matrix $A$ for type 1 methods, which are sequential explicit methods, is strictly lower triangular. These methods are appropriate for the numerical solution of nonstiff and mildly stiff ODEs in a sequential computing environment. Construction of DIMSIMs for the numerical solution of ODEs has been investigated in literature such as $[18,19]$. We recall that DIMSIM (1.3) with $r=s=p, U=I_{s}$, and $V \mathrm{e}=\mathrm{e}$ has order $p$ and stage order $q=p$ if and only if $[12,14,15]$

$$
B=B_{0}-A B_{1}-V B_{2}+V A,
$$

where the matrices $B_{0}, B_{1}$, and $B_{2} \in \mathbb{R}^{s \times s}$ have entries

$$
\left(B_{0}\right)_{i, j}=\frac{\int_{0}^{1+c_{i}} \phi_{j}(x) d x}{\phi_{j}\left(c_{j}\right)}, \quad\left(B_{1}\right)_{i, j}=\frac{\phi_{j}\left(1+c_{i}\right)}{\phi_{j}\left(c_{j}\right)}, \quad\left(B_{2}\right)_{i, j}=\frac{\int_{0}^{c_{i}} \phi_{j}(x) d x}{\phi_{j}\left(c_{j}\right)} .
$$

Here, $I_{s}$ stands for the identity matrix of dimension $s$, e is the $r$-dimensional all-ones vector, and $\phi_{i}(x)=\prod_{j=1, j \neq i}^{s}\left(x-c_{j}\right), i=1,2, \ldots, s$. In the linear stability analysis of the DIMSIMs for the test equation $y^{\prime}=\zeta y$, it is said that the method possesses Runge-Kutta stability (RKS) property if its stability function defined by

$$
p(\omega, z)=\operatorname{det}\left(\omega I_{r}-M(z)\right), \quad z:=\zeta h \in \mathbb{C},
$$

in which $M(z)=V+z B\left(I_{s}-z A\right)^{-1} U$ is the stability matrix of the method, has the special form

$$
p(\omega, z)=\omega^{r-1}(\omega-R(z)) .
$$

Combination of special DIMSIMs for ODEs with Gregory quadrature rule for solving (1.1), referred to GLMG, has been introduced in [31] which is reviewed in Section 2. The applied DIMSIMs in [31] are implicit so that their high accuracy and good stability properties cause the constructed such GLMG to be appropriate for solving stiff problems. In order to save computational effort in the case of non-stiff problems, however, explicit algorithms is preferred. Considering this point, in Section 3, we construct explicit GLMG schemes based on explicit DIMSIMs for ODEs with and without RKS property in which the free parameters of the methods are used to have RKS property for DIMSIMs or to extend the absolute stability region of DIMSIMs with the aim of maximizing the its area. The used strategy to achieve the maximum value for area is the same as that in $[2,3,5,6,23,24,25,28]$. Verification of the theoretical results, efficiency, and capability of the constructed methods in solving nonstiff and mildly stiff VIDEs together with comparison with implicit GLMG are given in Section 4. Finally, in Section 5 some concluding remarks are given. 


\section{A review on the GLMG}

We recall the GLMG schemes based on DIMSIMs of order $p$ and stage order $q=p$ together with Gregory quadrature rule for the numerical solution of VIDEs (1.1) which is defined by

$$
\left\{\begin{array}{l}
Y_{i}^{[n]}=h \sum_{j=1}^{s} a_{i j} f\left(t_{n-1}+c_{j} h, Y_{j}^{[n]}, Z_{j}^{[n]}\right)+\sum_{j=1}^{r} u_{i j} y_{j}^{[n-1]}, \quad i=1,2, \ldots, s, \\
y_{i}^{[n]}=h \sum_{j=1}^{s} b_{i j} f\left(t_{n-1}+c_{j} h, Y_{j}^{[n]}, Z_{j}^{[n]}\right)+\sum_{j=1}^{r} v_{i j} y_{j}^{[n-1]}, \quad i=1,2, \ldots, r
\end{array}\right.
$$

where $Z^{[n]}=\left[Z_{j}^{[n]}\right]_{j=1}^{s}$ is an approximation to the vector $z\left(t_{n-1}+c h\right)=$ $\left[z\left(t_{n-1}+c_{j} h\right)\right]_{j=1}^{s}$. The components of this vector are computed by Gregory quadrature rule of order $d$ such as

$$
\begin{aligned}
Z_{i}^{[n]}= & \frac{h}{s} \sum_{l=1}^{n-1} \sum_{j=1}^{s} w_{i j}^{(n, l)} K\left(t_{n-1}+c_{i} h, t_{l-1}+c_{j} h, Y_{j}^{[l]}\right) \\
& +\frac{h}{s} \sum_{j=1}^{i} w_{i j}^{(n, n)} K\left(t_{n-1}+c_{i} h, t_{n-1}+c_{j} h, Y_{j}^{[n]}\right), \quad i=1,2, \ldots, s,
\end{aligned}
$$

where $w_{i j}^{(n, l)}$ denote the weights of Gregory quadrature rule corresponding to abscissa $t_{l-1}+c_{j} h$. The coefficients matrices $A, U, B$, and $V$ in (2.1) are the same coefficients matrices of the constructed DIMSIMs with the abscissa vector $c$ of values equally spaced in the interval $[0,1]$ such that $c_{i}=(i-1) / s, i=$ $1,2, \ldots, s$. In [31], it is proved that the method (2.1) is convergent of order $\min \left\{p_{0}, p, d\right\}$, where $p_{0}$ is the order of the starting procedure for computing $Y^{[1]}$ and $y^{[1]}$. Also, the linear stability analysis of method (2.1) with respect to the basic test problem [9]

$$
\left\{\begin{array}{l}
y^{\prime}(t)=\gamma y(t)+\lambda \int_{0}^{t} y(s) d s, \quad t \geq 0, \\
y(0)=1,
\end{array}\right.
$$

with $\gamma$ and $\lambda$ as real parameters, is investigated in [31]. It is shown that the stability properties of these methods are governed by the stability matrix defined by

$$
\begin{aligned}
& M(\xi, \eta)= \\
& {\left[\begin{array}{ccc}
I_{s}-\xi A-\frac{\eta}{s} A L^{(n, n)} & 0 & 0 \\
-\xi B-\frac{\eta}{s} B L^{(n, n)} & I_{r} & 0 \\
-L^{(n+1, n)} & 0 & I_{s}
\end{array}\right]^{-1}\left[\begin{array}{ccc}
0 & U & \frac{\eta}{s} A \\
L^{(n+1, n-1)}-L^{(n, n-1)} & 0 & I_{s}
\end{array}\right]}
\end{aligned}
$$


with $\xi:=h \gamma, \eta:=h^{2} \lambda$, and for $l=1,2, \ldots, n-1$,

$$
\begin{aligned}
L^{(n, l)}:= & {\left[\begin{array}{cccc}
w_{11}^{(n, l)} & w_{12}^{(n, l)} & \cdots & w_{1 s}^{(n, l)} \\
w_{21}^{(n, l)} & w_{22}^{(n, l)} & \cdots & w_{2 s}^{(n, l)} \\
\vdots & \vdots & \ddots & \vdots \\
w_{s 1}^{(n, l)} & w_{s 2}^{(n, l)} & \cdots & w_{s s}^{(n, l)}
\end{array}\right], } \\
L^{(n, n)}:= & {\left[\begin{array}{cccc}
w_{11}^{(n, n)} & 0 & \cdots & 0 \\
w_{21}^{(n, n)} & w_{22}^{(n, n)} & \cdots & 0 \\
\vdots & \vdots & \ddots & \vdots \\
w_{s 1}^{(n, n)} & w_{s 2}^{(n, n)} & \cdots & w_{s s}^{(n, n)}
\end{array}\right] . }
\end{aligned}
$$

Then the corresponding stability function $p(\omega, \xi, \eta)$ is defined as the characteristic polynomial of $M(\xi, \eta)$, i.e.,

$$
p(\omega, \xi, \eta)=\operatorname{det}\left(\omega I_{2 s+r}-M(\xi, \eta)\right) .
$$

The absolute stability region $\mathcal{R}$ of method $(2.1)$ is a subset of the $(\xi, \eta)$-plane in which for every point in it, all the roots $\omega_{i}=\omega_{i}(\xi, \eta), i=1,2, \ldots, 2 s+r$, of $p(\omega, \xi, \eta)$ lie inside the unit circle with only simple roots on the boundary.

\section{Construction of the explicit GLMG with large regions of absolute stability}

In this section, we are going to construct explicit methods in the form (2.1) which is based on DIMSIM of type 1 of orders 2, 3, and 4 . We will refer to these methods as EGLMG.

In the construction of EGLMG, we consider two different cases: EGLMG based on DIMSIMs with and without RKS property. Indeed, in the construction DIMSIMs for EGLMG, after applying order and stage order conditions, a number of coefficients of the method remain as the free parameters; these free parameters are used for two aims: equipping DIMSIMs by RKS property or having a large stability region for DIMSIMs. The former needs to solve nonlinear algebraic equations [28], the latter can be done in some ways: to do this, we first define the objective function for the approximation of negative values of the area of the stability region as

$$
S:=-\frac{\Delta \theta}{2}\left(r^{2}\left(\theta_{0}\right)+2 \sum_{k=1}^{N-1} r^{2}\left(\theta_{k}\right)+r^{2}\left(\theta_{N}\right)\right),
$$

where $N$ is a positive integer and $\theta_{k}=k \Delta \theta, k=0,1, \ldots, N, N \Delta \theta=\pi / 2$. The rays $r_{k}=r\left(\theta_{k}\right), k=0,1, \ldots, N$, are computed by the bisection method applied to the equation

$$
p\left(w,-r_{k} \cos \left(\theta_{k}\right)+i r_{k} \sin \left(\theta_{k}\right)\right)=0,
$$

with $|w|=1$ and the stability function $p(w, z)$ defined by (1.5), which corresponds to the point on the boundary of the stability region. Then we use 
the fminsearch command of MATLAB to minimize this function (for more details see $[2,3])$.

In the construction of the methods, we assume that $p=q=r=s, U=I_{s}$, and $V=\mathrm{e} v^{T}$, and $v^{T} \mathrm{e}=1$. This representation of $V$ ensures zero-stability of the methods. According to the order conditions (1.4), the coefficient matrix $B$ depends only on the coefficient matrices $A$ and $V$. Hence, the only free parameters are the $s(s-1) / 2$ entries of the matrix $A$ and the $s-1$ entries of the matrix $V$. These free parameters are used to achieve the above-mentioned aims.

\subsection{Methods of order 2}

We start with the construction of DIMSIMs of type 1 with $p=q=r=s=2$ and $c=\left[\begin{array}{ll}0 & \frac{1}{2}\end{array}\right]^{T}$. By the order conditions (1.4), we obtain a two-parameter family of the methods depending on the parameters $a_{21}$ and $v_{1}$. The coefficient matrices of these methods are

$$
\begin{aligned}
& A=\left[\begin{array}{rr}
0 & 0 \\
a_{21} & 0
\end{array}\right], \quad B=\left[\begin{array}{cc}
-a_{21} v_{1}+a_{21}+0.25 v_{1}-0.25 & 0.25 v_{1}+0.75 \\
-a_{21} v_{1}+2 a_{21}+0.25 v_{1}-1 & -2 a_{21}+0.25 v_{1}
\end{array}\right], \\
& v=\left[\begin{array}{ll}
v_{1} & 1-v_{1}
\end{array}\right]^{T} .
\end{aligned}
$$

The stability polynomial $p(\omega, z)$ is given by

$$
p(\omega, z)=\omega^{2}+p_{1}(z) \omega+p_{0}(z),
$$

with

$$
\begin{aligned}
& p_{1}(z)=\left(-0.25 a_{21} v_{1}-0.75 a_{21}\right) z^{2}+\left(a_{21} v_{1}+a_{21}-0.5 v_{1}-1.75\right) z-1, \\
& p_{0}(z)=\left(0.5 v_{1}+0.25-0.25 a_{21}-0.75 a_{21} v_{1}\right) z^{2}+\left(0.5 v_{1}-a_{21}-a_{21} v_{1}+0.75\right) z .
\end{aligned}
$$

There is no such methods with RKS property. Now, by using the two free parameters and the mentioned numerical optimization procedure, we find the method with a large stability region with coefficients matrices as

$$
\begin{aligned}
& A=\left[\begin{array}{cc}
0 & 0 \\
0.477396064566154 & 0
\end{array}\right] \\
& B=\left[\begin{array}{ll}
0.338730045473526 & 0.627599046052331 \\
0.066126110039680 & 0.922806916920023
\end{array}\right], \\
& v=\left[\begin{array}{lll}
-0.489603815790677 & 1.489603815790677
\end{array}\right]^{T} .
\end{aligned}
$$

The stability region of the constructed DIMSIM has been plotted in Figure 1. Then the area of the stability region of the corresponding EGLMG with $d=2$ is approximately 19.78. This region has been plotted in Figure 2. To compare, we have also plotted the stability region of explicit Runge-Kutta method of order 2

\begin{tabular}{c|cc}
0 & 0 & 0 \\
1 & 1 & 0 \\
\hline & 0.5 & 0.5
\end{tabular}


combined with Gregory quadrature rule with $d=2$ (ERKG2).

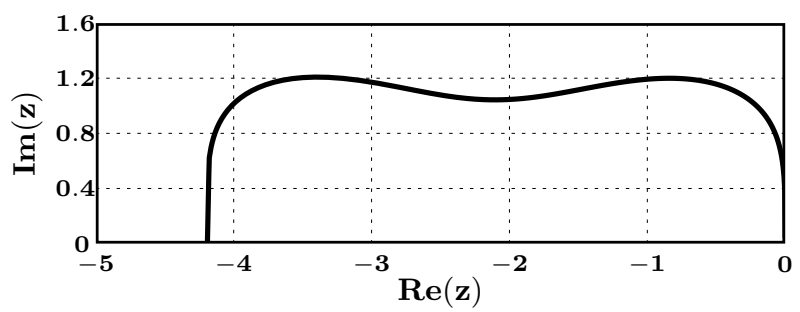

Figure 1. Region of absolute stability of the constructed DIMSIM without RKS property for $p=q=r=s=2$.

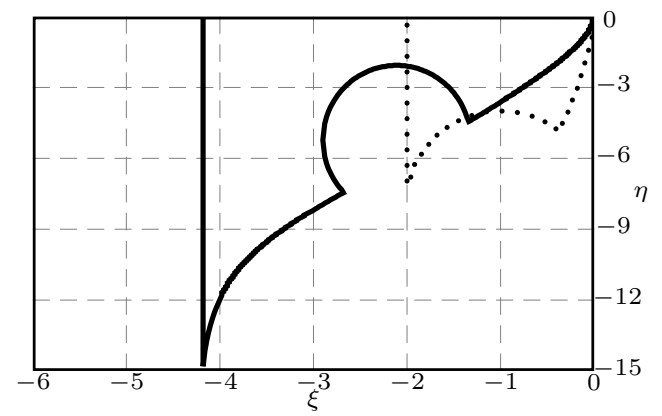

Figure 2. The absolute stability region of the EGLMG for $p=q=r=s=d=2$ (solid-line) together with that of ERKG2 (dotted-line).

\subsection{Methods of order 3}

In this subsection, we construct DIMSIMs of type 1 with $p=q=r=s=3$ and $c=\left[\begin{array}{lll}0 & \frac{1}{3} & \frac{2}{3}\end{array}\right]^{T}$. By the order conditions (1.4), we obtain a five-parameter family of the methods depending on the parameters $a_{21}, a_{31}, a_{32}, v_{1}$, and $v_{2}$. The construction of DIMSIM with RKS property leads to

$$
\begin{aligned}
& a_{21}=0.364579395708913, \quad v_{1}=0.278913079293709, \\
& a_{31}=-1.340524014973630, \quad v_{2}=-1.130081668799246, \\
& a_{32}=0.891259309962354
\end{aligned}
$$

Also, searching for these free parameters to construct DIMSIM with a maximum stability region leads to DIMSIM with the following coefficients matrices

$$
\begin{aligned}
A & =\left[\begin{array}{ccc}
0 & 0 & 0 \\
0.427348649099458 & 0 & 0 \\
0.403774175393196 & 0.416387121220301 & 0
\end{array}\right], \\
B & =\left[\begin{array}{lcc}
0.414277043879229 & 0.259957591597852 & 0.493084210189841 \\
0.625817283668660 & -0.235774238881552 & 0.683260485113690 \\
0.872452615936241 & -0.753178468015707 & 0.894550067799558
\end{array}\right],
\end{aligned}
$$




$$
v=\left[\begin{array}{lll}
0.459033630195495 & -1.417020590789537 & 1.957986960594042
\end{array}\right]^{T} .
$$

The stability regions of the constructed DIMSIMs with and without RKS property have been plotted in Figure 3. Then the area of the stability region of the corresponding EGLMG with $d=3$ is approximately 48.15. This area, as it can be seen in Figure 4, is much larger than that based on DIMSIM with RKS property. Also, to compare, we have plotted the stability region of explicit Runge-Kutta method of order 3

\begin{tabular}{c|ccc}
0 & 0 & 0 & 0 \\
$\frac{1}{2}$ & $\frac{1}{2}$ & 0 & 0 \\
1 & -1 & 2 & 0 \\
\hline & $\frac{1}{6}$ & $\frac{2}{3}$ & $\frac{1}{6}$
\end{tabular}

combined with Gregory quadrature rule with $d=3$ (ERKG3).

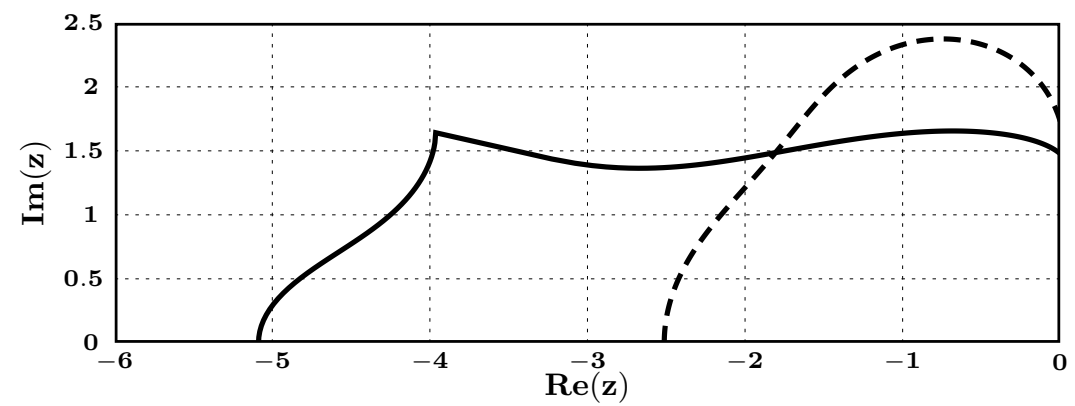

Figure 3. Regions of absolute stability of the constructed DIMSIMs with (dashed-line) and without (solid-line) RKS property for $p=q=r=s=3$.

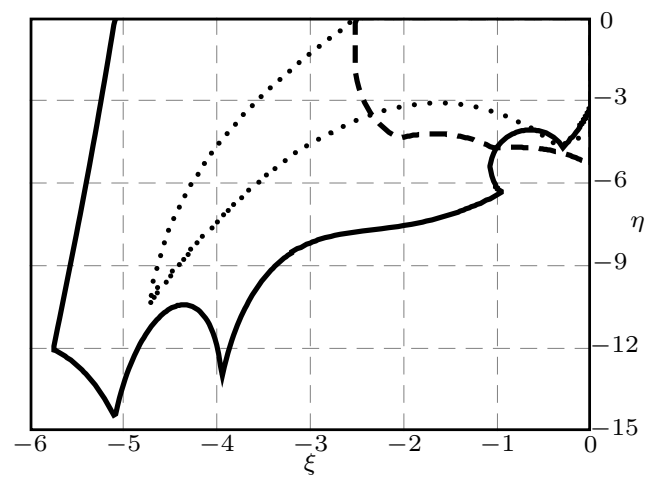

Figure 4. The absolute stability region of the EGLMG based on DIMSIM with (dashed-line) and without (solid-line) RKS property for $p=q=r=s=d=3$ together with that of ERKG3 (dotted-line).

\subsection{Methods of order 4}

In this subsection, we construct DIMSIMs of type 1 with $p=q=r=s=4$ and $c=\left[\begin{array}{cccc}0 & \frac{1}{4} & \frac{2}{4} & \frac{3}{4}\end{array}\right]^{T}$. By the order conditions (1.4), we obtain a nine-parameter 
family of the methods depending on the parameters $a_{21}, a_{31}, a_{32}, a_{41}, a_{42}, a_{43}$, $v_{1}, v_{2}$, and $v_{3}$. The construction of DIMSIM with RKS property leads to

$$
\begin{aligned}
& a_{21}=-0.918469950042997, \quad v_{1}=-0.310385932460329, \\
& a_{31}=1.891928646383971, \quad v_{2}=1.511553585168678, \\
& a_{32}=-0.204196152762772, \quad v_{3}=-2.910769407345565, \\
& a_{41}=2.063950266436812, \quad a_{42}=-0.724353911408417, \\
& a_{43}=0.559756779666624,
\end{aligned}
$$

Again, searching for these free parameters to construct DIMSIM with a maximum stability region leads to DIMSIM with the following coefficients matrices

$$
\begin{aligned}
A & =\left[\begin{array}{cccc}
0 & 0 & 0 & 0 \\
0.217726256805536 & 0 & 0 & 0 \\
-0.312422961871620 & 0.445993427959222 & 0 & 0 \\
-0.462182301094931 & 0.369893002597912 & 0.362246549335681 & 0
\end{array}\right], \\
B & =\left[\begin{array}{llll}
-0.72716469319 & 0.82959968202 & -0.21857408420 & 0.53925480109 \\
-1.08235510305 & 2.15661132146 & -1.96429987670 & 1.34543310720 \\
-1.50561394323 & 3.72272344345 & -4.08990996292 & 2.66234570232 \\
-1.99355949054 & 5.37030807134 & -6.01146311871 & 3.53787299278
\end{array}\right], \\
v & =\left[\begin{array}{llll}
0.15584949591 & -0.32630377439 & -0.22486159584 & 1.39531587433
\end{array}\right]^{T} .
\end{aligned}
$$

The stability regions of the constructed DIMSIMs with and without RKS property have been plotted in Figure 5. Then the area of the stability region of the corresponding EGLMG with $d=4$ is approximately 45.44 which is, as shown in Figure 6, much larger than that of the method based on DIMSIM with RKS property. To compare, we have also plotted the stability region of explicit Runge-Kutta method of order 4

\begin{tabular}{c|cccc}
0 & 0 & 0 & 0 & 0 \\
$\frac{1}{2}$ & $\frac{1}{2}$ & 0 & 0 & 0 \\
$\frac{1}{2}$ & 0 & $\frac{1}{2}$ & 0 & 0 \\
1 & 0 & 0 & 1 & 0 \\
\hline & $\frac{1}{6}$ & $\frac{1}{3}$ & $\frac{1}{3}$ & $\frac{1}{6}$
\end{tabular}

combined with Gregory quadrature rule with $d=4$ (ERKG4).

\section{Numerical verifications}

In this section, we present numerical results showing the efficiency and accuracy of the constructed EGLMG, validating the order of these methods in the integration of nonstiff VIDEs, and comparing the results with those of implicit GLMG proposed in [31]. In our implementation, we use the starting procedure proposed in [31] to compute the necessary starting values which we recall it here: the starting vector $y^{[1]}$ can be computed by the values $Y_{i}^{[1]}, i=2,3, \ldots, s$, and 


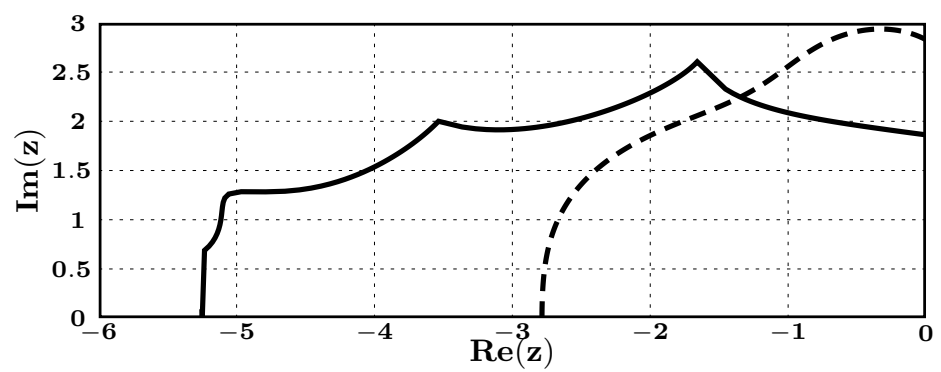

Figure 5. Regions of absolute stability of the constructed DIMSIMs with (dashed-line) and without (solid-line) RKS property for $p=q=r=s=4$.

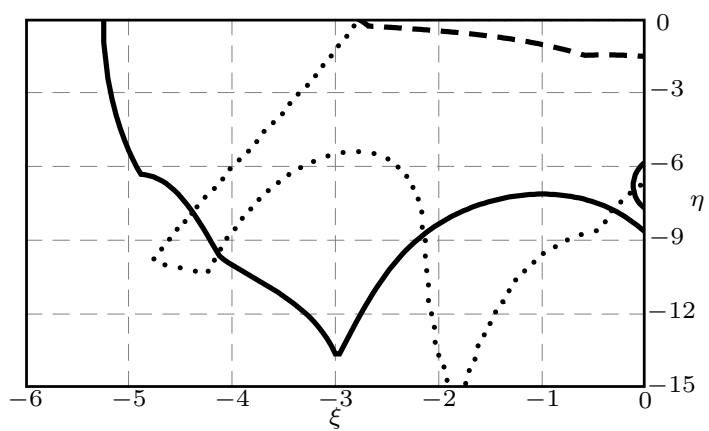

Figure 6. The absolute stability region of the EGLMG based on DIMSIM with (dashed-line) and without (solid-line) RKS property for $p=q=r=s=d=4$ together with that of ERKG4 (dotted-line).

$y_{1}$ which should be obtained simultaneously to be of the same order of the method. These values can be computed as

$$
\left\{\begin{array}{l}
\sum_{j=1}^{s} \widehat{a}_{i j} Y_{j}^{[1]}+\widehat{a}_{i, s+1} y_{1}=h f\left(t_{0}+c_{i} h, Y_{i}^{[1]}, Z_{i}^{[1]}\right), \quad i=2,3, \ldots, s, \\
\sum_{j=1}^{s} \widehat{a}_{s+1, j} Y_{j}^{[1]}+\widehat{a}_{s+1, s+1} y_{1}=h f\left(t_{1}, y_{1}, Z^{[1]}\right),
\end{array}\right.
$$

where

$$
\begin{aligned}
& Z_{i}^{[1]}=h \sum_{j=1}^{s} \widehat{b}_{i j} K\left(t_{0}+c_{i} h, t_{0}+c_{j} h, Y_{j}^{[1]}\right)+h \widehat{b}_{i, s+1} K\left(t_{0}+c_{i} h, t_{1}, y_{1}\right), i=2,3, \ldots, s, \\
& Z^{[1]}=h \sum_{j=1}^{s} \widehat{b}_{s+1, j} K\left(t_{1}, t_{0}+c_{j} h, Y_{j}^{[1]}\right)+h \widehat{b}_{s+1, s+1} K\left(t_{1}, t_{1}, y_{1}\right) .
\end{aligned}
$$

The values for the coefficients $\widehat{a}_{i j}$, for $i=2,3, \ldots, s+1$ and $j=1,2, \ldots, s+1$ are given in [31]. Then $y^{[1]}$ is computed by

$$
y^{[1]}=\left(Q T^{-1} \otimes I_{m}\right)\left[\begin{array}{lllll}
Y_{1} & Y_{2} & \cdots & Y_{s} & y_{1}
\end{array}\right]^{T},
$$


where $Q=\left[\begin{array}{llll}q_{0} & q_{1} & \cdots & q_{s}\end{array}\right]$ and $T=\left[t_{i, j}\right]$ is an $(s+1) \times(s+1)$ matrix given by

$$
t_{i, j}=\left\{\begin{array}{cl}
\frac{\left(c_{i}-1\right)^{j-1}}{(j-1) !}, & i \neq s+1 \\
\delta_{1 j}, & i=s+1
\end{array}\right.
$$

where $\delta_{1 j}$ denotes the Kronecker delta.

To show the effect of the stability region of the methods, we consider the linear VIDE [20]

$$
\left\{\begin{array}{l}
y^{\prime}(t)=\gamma(y(t)-\sin (t))+1-\int_{0}^{t} y(s) d s, \quad t \in[0,10] \\
y(0)=0
\end{array}\right.
$$

with $\gamma<0$ and exact solution $y(t)=\sin (t)$. This problem is equivalent to a system of ODEs of Prothero-Robinson type with eigenvalues $\gamma_{1}, \gamma_{2}$ in which $\frac{\left|\gamma_{1}\right|}{\left|\gamma_{2}\right|}=\mathcal{O}\left(\gamma^{2}\right)$ is the stiffness ratio. We applied EGLMG of order three with and without RKS property on this problem for $\gamma=-20$ with fixed stepsizes $h_{1}=0.2$ and $h_{2}=0.1$. Considering the absolute stability regions in Figure 4 , $\left(-20 h_{1},-h_{1}^{2}\right)$ lies outside of the stability region for EGLMG with RKS while it lies inside of this region for EGLMG without RKS. However, $\left(-20 h_{2},-h_{2}^{2}\right)$ lies inside of the stability region for both of them. In Figure 7, we have plotted the error of the methods over whole of the interval with different stepsizes $h_{1}$ and $h_{2}$. In comparison EGLMG with and without RKS property, as we expect, for $h=h_{1}$, the results show that the absolute instability of the former manifests itself in the form of a violently growing error, whereas the latter remains stable and accurate.
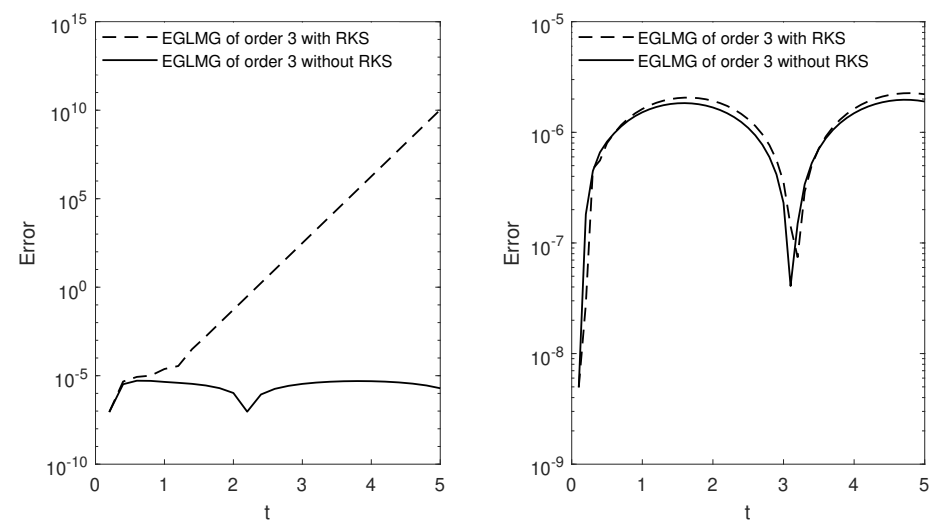

Figure 7. Errors versus $t$ for EGLMG of order 3 applied to problem (4.1) for fixed stepsizes $h_{1}=0.2$ (left) and $h_{2}=0.1$ (right).

To illustrate the accuracy of the proposed methods, we apply them to the 
linear VIDE $[4,27,29,36]$

$$
\left\{\begin{array}{l}
y^{\prime}(t)=1+2 t-y(t)+\int_{0}^{t} t(1+2 t) e^{s(t-s)} y(s) d s, \quad t \in[0,1] \\
y(0)=1
\end{array}\right.
$$

with the exact solution $y(t)=e^{t^{2}}$. We have implemented the methods with a fixed stepsize $h=\frac{1}{2^{k}}$ with several integer values of $k$. Table 1 shows the errors $e_{N}(h)$ at the endpoint of the interval of integration, and the numerical estimation for the order of convergence of the methods computed by $p:=$ $\log _{2}\left(e_{N}(h) / e_{N}(h / 2)\right)$. The results show the high accuracy of the methods and confirm their theoretical orders.

Table 1. Numerical results of EGLMG without RKS property of orders 2, 3, and 4 for problem (4.2).

\begin{tabular}{|c|c|c|c|c|c|c|}
\hline \multirow{2}{*}{$k$} & \multicolumn{2}{|c|}{ Order 2 method } & \multicolumn{2}{|c|}{ Order 3 method } & \multicolumn{2}{|c|}{ Order 4 method } \\
\hline & $e_{N}(h)$ & $p$ & $e_{N}(h)$ & $p$ & $e_{N}(h)$ & $p$ \\
\hline 4 & $1.02 \times 10^{-2}$ & & $5.49 \times 10^{-4}$ & & $2.24 \times 10^{-5}$ & \\
\hline 5 & $2.74 \times 10^{-3}$ & 1.89 & $7.51 \times 10^{-5}$ & 2.87 & $1.58 \times 10^{-6}$ & 3.83 \\
\hline 6 & $7.10 \times 10^{-4}$ & 1.95 & $9.83 \times 10^{-6}$ & 2.93 & $1.05 \times 10^{-7}$ & 3.91 \\
\hline 7 & $1.81 \times 10^{-4}$ & 1.97 & $1.26 \times 10^{-6}$ & 2.97 & $6.75 \times 10^{-9}$ & 3.96 \\
\hline 8 & $4.56 \times 10^{-5}$ & 1.99 & $1.59 \times 10^{-7}$ & 2.98 & $4.29 \times 10^{-10}$ & 3.98 \\
\hline 9 & $1.15 \times 10^{-5}$ & 1.99 & $2.00 \times 10^{-8}$ & 2.99 & $2.70 \times 10^{-11}$ & 3.99 \\
\hline
\end{tabular}

Next we consider the nonlinear VIDE $[9,34,37]$

$$
\left\{\begin{array}{l}
y^{\prime}(t)=-\frac{1+t(1+t)^{2}}{(1+t)^{2}}+\frac{1}{y(t)} \ln \left(\frac{2+2 t}{2+t}\right)+\int_{0}^{t} \frac{d s}{1+(1+t) y(s)}, \quad t \in[0,10] \\
y(0)=1
\end{array}\right.
$$

with the exact solution $y(t)=\frac{1}{1+t}$. In Table 2 , we have repeated the numerical results reported in Table 1 but now for example (4.3). The results in this table show that the errors decrease with the expected orders 2,3 , and 4 .

As the last problem, we consider

$$
\left\{\begin{array}{l}
y^{\prime}(t)=1-t e^{-t^{2}}+y(t)-2 \int_{0}^{t} t s e^{-y(s)^{2}} d s, \quad t \in[0,10] \\
y(0)=0
\end{array}\right.
$$

with the exact solution $y(t)=t$. In Figure 8, we have compared the results of (implicit) GLMG given in [31] and (explicit) EGLMG constructed in Section 3 of orders 3 and 4 . In this figure, we have plotted the number of kernel evaluations versus the accuracy of the methods. Although this figure shows a small improvement of proposed explicit methods comparing with the implicit 
Table 2. Numerical results of EGLMG without RKS property of orders 2, 3, and 4 for problem (4.3).

\begin{tabular}{|c|c|c|c|c|c|c|}
\hline \multirow{2}{*}{$k$} & \multicolumn{2}{|c|}{ Order 2 method } & \multicolumn{2}{|c|}{ Order 3 method } & \multicolumn{2}{|c|}{ Order 4 method } \\
\hline & $e_{N}(h)$ & $p$ & $e_{N}(h)$ & $p$ & $e_{N}(h)$ & $p$ \\
\hline 4 & $1.56 \times 10^{-6}$ & & $6.18 \times 10^{-8}$ & & $2.90 \times 10^{-9}$ & \\
\hline 5 & $4.05 \times 10^{-7}$ & 1.95 & $8.51 \times 10^{-9}$ & 2.86 & $2.02 \times 10^{-10}$ & 3.84 \\
\hline 6 & $2.64 \times 10^{-8}$ & 1.96 & $1.12 \times 10^{-9}$ & 2.93 & $1.34 \times 10^{-11}$ & 3.92 \\
\hline 7 & $2.64 \times 10^{-8}$ & 1.98 & $1.43 \times 10^{-10}$ & 2.96 & $8.59 \times 10^{-13}$ & 3.96 \\
\hline 8 & $6.64 \times 10^{-9}$ & 1.99 & $1.81 \times 10^{-11}$ & 2.98 & $5.44 \times 10^{-14}$ & 3.98 \\
\hline 9 & $1.67 \times 10^{-9}$ & 1.99 & $2.28 \times 10^{-12}$ & 2.99 & $3.43 \times 10^{-15}$ & 3.99 \\
\hline
\end{tabular}

ones, considering the required number of Jacobian evaluations together with the solving linear systems, the proposed methods outperform the implicit GLMG for nonstiff problems for more stringent tolerances.

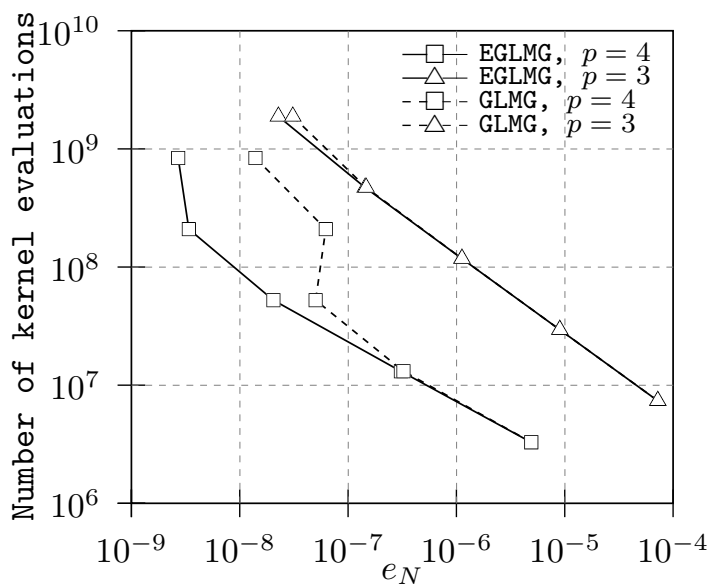

Figure 8. The number of kernel evaluations versus global error at the end point $T=10$ for problem (4.4).

\section{Conclusions}

To construct explicit algorithm for the numerical solution of VIDEs, we combined ODE solver DIMSIMs of type 1 with Gregory quadrature rule which was referred to EGLMG. This method, indeed, is actually the explicit form of GLMG introduced in [31]. Methods of this class of order 2 without RKS property and of orders 3 and 4 in both cases with and without RKS property were constructed. Using the free parameters in the methods without RKS property, we constructed methods with a large region of absolute stability regions which can successfully solve VIDEs with large stepsizes than those with RKS property of the same order. Theoretical results, efficiency and accuracy 
of the constructed methods were verified by some numerical experiments. Furthermore, comparing with implicit GLMG, the results confirmed that EGLMG could be more efficient for nonstiff VIDEs.

\section{Acknowledgements}

The work of this paper was supported by the University of Tabriz, Iran under research grant No. 816.

\section{References}

[1] A. Abdi and S.A. Hosseini. The barycentric rational differencequadrature scheme for systems of Volterra integro-differential equations. SIAM Journal on Scientific Computing, 40(3):A1936-A1960, 2018. https://doi.org/10.1137/17M114371X.

[2] N. Barghi Oskouie, G. Hojjati and A. Abdi. Efficient second derivative methods with extended stability regions for non-stiff IVPs. Computational and Applied Mathematics, 37(2):2001-2016, 2018. https://doi.org/10.1007/s40314-017-0436-y.

[3] Z. Bartoszewski and Z. Jackiewicz. Explicit Nordsieck methods with extended stability regions. Applied Mathematics and Computation, 218(10):6056-6066, 2012. https://doi.org/10.1016/j.amc.2011.11.088.

[4] M.I. Berenguer, M.A. Fortes, A.I. Guillem-Garralda and M.R. Galán. Linear Volterra integro-differential equation and Schauder bases. Applied Mathematics and Computation, 159(2):495-507, 2004. https://doi.org/10.1016/j.amc.2003.08.132.

[5] M. Braś and A. Cardone. Construction of efficient general linear methods for non-stiff differential systems. Mathematical Modelling and Analysis, 17(2):171189, 2012. https://doi.org/10.3846/13926292.2012.655789.

[6] M. Braś, A. Cardone and R. D'Ambrosio. Implementation of explicit Nordsieck methods with inherent quadratic stability. Mathematical Modelling and Analysis, 18(2):289-307, 2013. https://doi.org/10.3846/13926292.2013.785039.

[7] H. Brunner. Implicit Runge-Kutta methods of optimal order for Volterra integrodifferential equations. Mathematics of Computation, 42(165):95-109, 1984. https://doi.org/10.2307/2007561.

[8] H. Brunner. Collocation methods for Volterra integral and related functional differential equations, volume 15. Cambridge University Press, 2004.

[9] H. Brunner and J.D. Lambert. Stability of numerical methods for Volterra integro-differential equations. Computing, 12(1):75-89, 1974. https://doi.org/10.1007/BF02239501.

[10] H. Brunner and P.J. van der Houwen. The numerical solution of Volterra equations, volume 3. Elsevier Science Ltd, 1986.

[11] J.C. Butcher. On the convergence of numerical solutions to ordinary differential equations. Math. Comp., 20(93):1-10, 1966. https://doi.org/10.1090/S0025-5718-1966-0189251-X.

[12] J.C. Butcher. Diagonally-implicit multi-stage integration methods. Applied Numerical Mathematics, 11(5):347-363, 1993. https://doi.org/10.1016/0168-9274(93)90059-Z. 
[13] J.C. Butcher. Numerical methods for ordinary differential equations. Wiley, 2016.

[14] J.C. Butcher and Z. Jackiewicz. Diagonally implicit general linear methods for ordinary differential equations. BIT Numerical Mathematics, 33(3):452-472, 1993. https://doi.org/10.1007/BF01990528.

[15] J.C. Butcher and Z. Jackiewicz. Construction of diagonally implicit general linear methods of type 1 and 2 for ordinary differential equations. Applied Numerical Mathematics, 21(4):385-415, 1996. https://doi.org/10.1016/S0168-9274(96)00043-8.

[16] J.C. Butcher and Z. Jackiewicz. Implementation of diagonally implicit multistage integration methods for ordinary differential equations. SIAM Journal on Numerical Analysis, 34(6):2119-2141, 1997. https://doi.org/10.1137/S0036142995282509.

[17] J.C. Butcher and Z. Jackiewicz. Construction of high order diagonally implicit multistage integration methods for ordinary differential equations. Applied Numerical Mathematics, 27(1):1-12, 1998. https://doi.org/10.1016/S0168-9274(97)00109-8.

[18] J.C. Butcher and Z. Jackiewicz. Construction of general linear methods with Runge-Kutta stability properties. Numer. Algor., 36(1):53-72, 2004. https://doi.org/10.1023/B:NUMA.0000027738.54515.50.

[19] J.C. Butcher and W.M. Wright. The construction of practical general linear methods. BIT, 43(4):695-721, 2003. https://doi.org/10.1023/B:BITN.0000009952.71388.23.

[20] A. Cardone and D. Conte. Multistep collocation methods for Volterra integrodifferential equations. Applied Mathematics and Computation, 221:770-785, 2013. https://doi.org/10.1016/j.amc.2013.07.012.

[21] A. Cardone, D. Conte, R. D'Ambrosio and B. Paternoster. Collocation methods for Volterra integral and integro-differential equations: A review. Axioms, 7(3):45, 2018. https://doi.org/10.3390/axioms7030045.

[22] A. Cardone, D. Conte and B. Paternoster. A family of multistep collocation methods for Volterra integro-differential equations. In AIP Conference Proceedings, volume 1168, pp. 358-361. AIP, 2009. https://doi.org/10.1063/1.3241469.

[23] A. Cardone and Z. Jackiewicz. Explicit Nordsieck methods with quadratic stability. Numer. Algor., 60(1):1-25, 2012. https://doi.org/10.1007/s11075-011-9509-y.

[24] A. Cardone, Z. Jackiewicz and H. Mittelmann. Optimization-based search for Nordsieck methods of high order with quadratic stability polynomials. Mathematical Modelling and Analysis, 17(3):293-308, 2012. https://doi.org/10.3846/13926292.2012.685497.

[25] J. Chollom and Z. Jackiewicz. Construction of two-step RungeKutta methods with large regions of absolute stability. Journal of Computational and Applied Mathematics, 157(1):125-137, 2003. https://doi.org/10.1016/S0377-0427(03)00382-0.

[26] D. Costarelli and R. Spigler. A collocation method for solving nonlinear Volterra integro-differential equations of neutral type by sigmoidal functions. Journal of Integral Equations and Applications, 26(1):15-52, 2014. https://doi.org/10.1216/JIE-2014-26-1-15. 
[27] S. Fazeli and G. Hojjati. Numerical solution of Volterra integro-differential equations by superimplicit multistep collocation methods. Numerical Algorithms, 68(4):741-768, 2015. https://doi.org/10.1007/s11075-014-9870-8.

[28] Z. Jackiewicz. General Linear Methods for Ordinary Differential Equations. John Wiley \& Sons, 2009.

[29] P. Linz. Analytical and numerical methods for Volterra equations, volume 7. SIAM, 1985.

[30] C. Lubich. Runge-Kutta theory for Volterra integrodifferential equations. Numerische Mathematik, 40(1):119-135, 1982. https://doi.org/10.1007/BF01459081.

[31] H. Mahdi, A. Abdi and G. Hojjati. Efficient general linear methods for a class of Volterra integro-differential equations. Applied Numerical Mathematics, 127:95109, 2018. https://doi.org/10.1016/j.apnum.2018.01.001.

[32] A. Makroglou. Hybrid methods in the numerical solution of Volterra integrodifferential equations. IMA Journal of Numerical Analysis, 2(1):21-35, 1982. https://doi.org/10.1093/imanum/2.1.21.

[33] J. Matthys. A-stable linear multistep methods for Volterra integrodifferential equations. Numerische Mathematik, 27(1):85-94, 1976. https://doi.org/10.1007/BF01399087.

[34] B.P. Sommeijer, W. Couzy and P.J. van der Houwen. A-stable parallel block methods for ordinary and integro-differential equations. Applied numerical mathematics, 9(3-5):267-281, 1992. https://doi.org/10.1016/0168-9274(92)90021-5.

[35] Y. Wei and Y. Chen. Legendre spectral collocation method for neutral and highorder Volterra integro-differential equation. Applied Numerical Mathematics, 81:15-29, 2014. https://doi.org/10.1016/j.apnum.2014.02.012.

[36] M.A. Wolfe and G.M. Phillips. Some methods for the solution of non-singular Volterra integro-differential equations. The Computer Journal, 11(3):334-336, 1968. https://doi.org/10.1093/comjnl/11.3.334.

[37] P.H.M. Wolkenfelt. The construction of reducible quadrature rules for Volterra integral and integro-differential equations. IMA Journal of Numerical Analysis, 2(2):131-152, 1982. https://doi.org/10.1093/imanum/2.2.131. 